\title{
COMPARISON OF TRACKING CODES FOR THE INTERNATIONAL LINEAR COLLIDER *
}

\author{
Jeffrey C. Smith ${ }^{\dagger}$, CLASSE, Cornell Univ., Ithaca, USA, Andrea Latina, Daniel Schulte, CERN, \\ Geneva, Switzerland, Freddy Poirier, Nicholas Walker, DESY, Hamburg, Germany, Paul Lebrun, \\ Kirti Ranjan, Fermilab, Batavia, USA, Kiyoshi Kubo, KEK, Ibaraki, Japan, Peter Tenenbaum, \\ SLAC, Menlo Park, USA, Peder Eliasson, Uppsala University, Uppsala, Sweden
}

\section{Abstract}

In an effort to compare beam dynamics and create a "benchmark" for Dispersion Free Steering (DFS) a comparison was made between different International Linear Collider (ILC) simulation programs while performing DFS. This study consisted of three parts. Firstly, a simple betatron oscillation was tracked through each code. Secondly, a set of component misalignments and corrector settings generated from one program was read into the others to confirm similar emittance dilution. Thirdly, given the same set of component misalignments, DFS was performed independently in each program and the resulting emittance dilution was compared. Performance was found to agree exceptionally well in all three studies.

\section{INTRODUCTION}

Comparisons of the performance between ILC simulations codes are well documented [1, 2, 3, 4]. The general conclusion is that BMAD [5], LIAR [6], Merlin [7], PLACET [8] and MAD [9] simulate the emittance dilution in the ILC main linac consistently. The purpose of this study is to extend the simulation crosschecking to include the explicit performance of a specific static BeamBased Alignment (BBA) algorithm. DFS was chosen as it is the most complex and widely used of the BBA methods. Details of the DFS method used can be found in [10]. Three new codes are now active in ILC Low Emittance Transport (LET) simulations CHEF [11], Lucretia [12] and SLEPT [13]. One of these new codes, SLEPT, has been included in the following analysis. It was decided that the TESLA TDR lattice would be the benchmarking lattice. The purpose of this study was not to determine the absolute alignment requirements or absolute DFS performance but rather to compare the simulation codes and DFS implementations so the choice of the lattice was rather arbitrary. Some codes track macroparticles (second moments) whereas others track a distribution of single particles (first moments). The number of particles tracked also varies between codes.

\footnotetext{
* Work supported by the US Department of Energy under contracts DEFG02-04ER41352 and DE-AC02-76SF00515, the US National Science Foundation under contract PHY-0202078 and the Commission of the European Communities under the 6th Framework Programme "Structuring the European Research Area", contract number RIDS-011899.

$\dagger$ js344@slac.stanford.edu
}

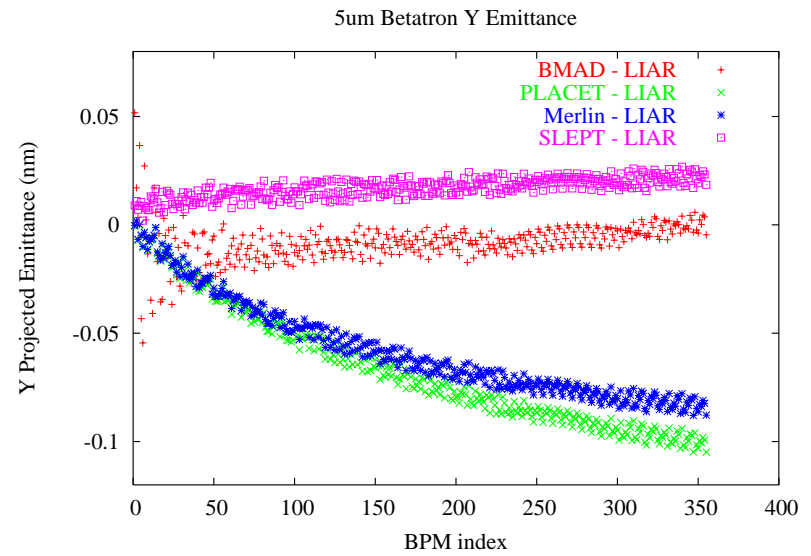

Figure 1: Betatron oscillation emittance growth through the main linac due to a 5 micron vertical offset. Plotted here is the difference in emittance between five codes.

\section{STUDY \#1: BETATRON OSCILLATION}

The first study tracked a betatron oscillation due to a 5 micron vertically offset beam entering the perfectly aligned linac. Figure 1 gives the difference in emittance for the five codes with respect to the LIAR results. For LIAR, the total emittance growth is $1.2 \mathrm{~nm}$ and the difference is small compared to the absolute emittance growth. Also, since the desired accuracy in emittance measurements need not be greater than $5 \%$ of $10 \mathrm{~nm}$, or $0.5 \mathrm{~nm}$, the variance in the results of no more than $0.1 \mathrm{~nm}$ is negligible.

This study led to the discovery of various differences between the codes. A wakefield lookup table was supplied with the lattice file to ensure every code used the same function. However, different codes use different methods to calculate the reference energy. Since the quadrupole strengths are relative to the reference energy, this was found to be an issue with the focusing optics. Specifically, due to beam loading, the cavities will have lower gradients than the design value of $23.4 \mathrm{MeV} / \mathrm{m}$. Different codes calculate the beam loss separately and a common beam loading value was used to to ensure consistency.

Another issue was related to the ponderomotive force. One code package, BMAD, includes this force in the accelerating cavities. The ponderomotive force was found to affect the orbit on the order of 0.1 microns in this study and affects the results on the scale of Figure 1. The pondero- 
motive force was turned off in BMAD for the remainder of the studies. The effect of the ponderomotive force on the emittance is discussed in more detail in [14].

Table 1: Main Linac RMS misalignments used in study.

\begin{tabular}{|l|r|r|}
\hline Error & RMS & With respect to... \\
\hline Quad Offset & $300 \mu \mathrm{m}$ & Cryostat \\
\hline Quad Tilt & $300 \mu \mathrm{rad}$ & Cryostat \\
\hline BPM Offset & $300 \mu \mathrm{m}$ & Cryostat \\
\hline RF Cavity Offset & $300 \mu \mathrm{m}$ & Cryostat \\
\hline RF Cavity Pitch & $200 \mu \mathrm{rad}$ & Cryostat \\
\hline Cryostat Offset & $200 \mu \mathrm{m}$ & Survey Line \\
\hline
\end{tabular}

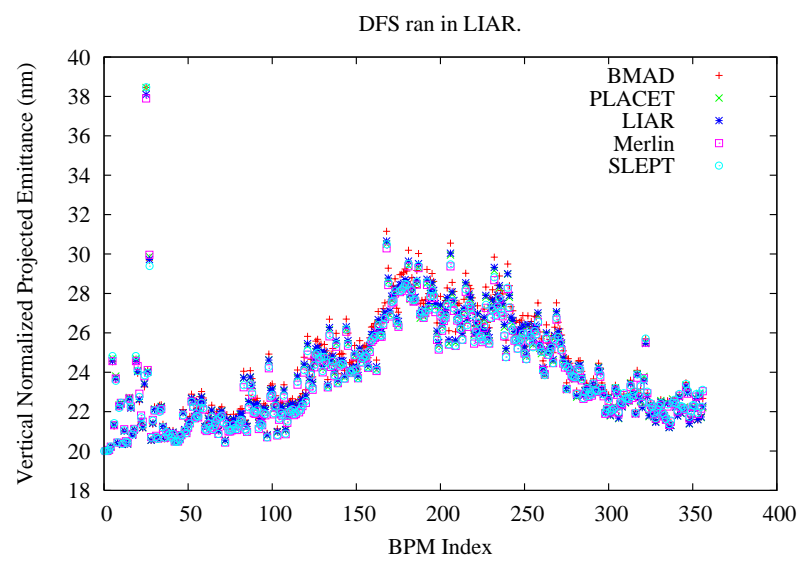

Figure 2: Emittance dilution after performing DFS in LIAR and reading the misalignments and corrector settings into the other codes.

\section{STUDY \#2: A STANDARD SET OF MISALIGNMENTS AND CORRECTOR SETTINGS}

The second study consisted of generating a set of misalignments and then running DFS in only one of the codes. This set of misalignments and corrector settings was then loaded into the other codes to compare the emittance dilution in each. Table 1 gives the RMS values used to generate the misalignment file. Figure 2 gives the emittance dilution in each of the codes after reading in the misalignments and corrector settings generated in LIAR. The agreement is remarkable and well within acceptable levels. This agreement was not achieved, however, without the discovery and correction of bugs in some of the codes. The spikes seen at the beginning of figure 2 are due to the DFS method used in LIAR and have since been eliminated. Nevertheless, the other codes reproduce the spikes obediently.

\section{STUDY \#3: A STANDARD SET OF MISALIGNMENTS BUT INDEPENDENT DFS RUNS}

The final study consisted of a single code generating a 100 seed set of misalignment files and then the other codes

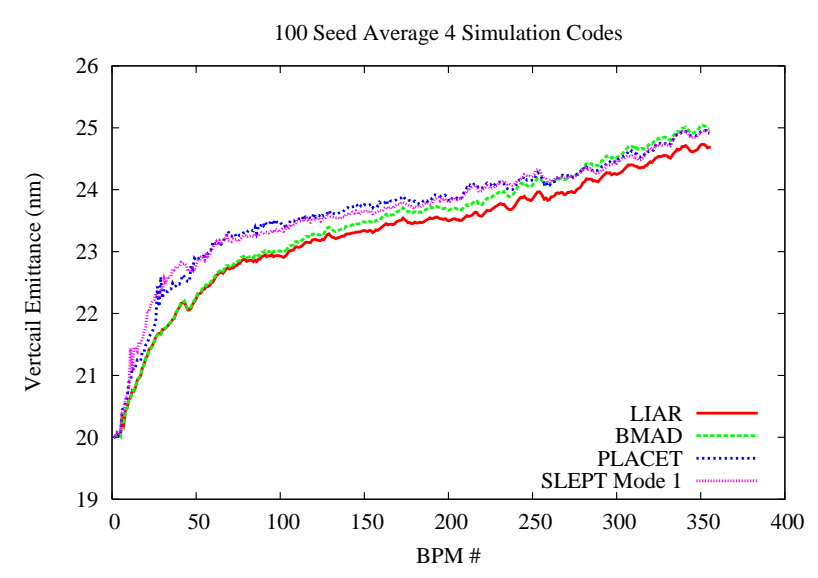

Figure 3: Each code performing DFS separately using the same 100 seeds.

reading in the misalignments and performing their own DFS on each. The various codes do make slight modifications to the DFS method. The BPM resolution was set to zero (i.e. perfect BPM resolution) in an effort to simplify the problem. To further simplify the problem, the first 9 cryomodules plus all components located on them were perfectly aligned. This was done in order to ignore the varying methods used to re-steer the beginning of the linac. DFS cannot be performed in this region due to insufficient energy variation from turned off cavities. Figure 3 gives the results of the study. The agreement is again very good. A separate study has been performed comparing PLACET to MERLIN using a separate lattice and misalignment seeds [15]. Agreement was again found to be quite good between these two codes and complements the work done in this study.

\section{Detailed Study Between BMAD and LIAR}

BMAD and LIAR have the most similar agreement in Figure 3. This is not a coincidence. These two teams spent an extended period of time comparing the performance of the two codes and were able to identify the key factors contributing to any differences between them. One notable difference was the method used to re-steer the offenergy beam. LIAR includes three steering magnets before each region in the optimization process whereas BMAD performs the steering as a separate step. The re-steering method in LIAR was changed to the BMAD method. This change was found to slightly degrade the performance in LIAR which suggests that it is advantageous to include the re-steering in the optimization rather than perform it separately. Another issue is precisely which accelerating cavities are turned off. It would be too laborious to describe precisely which cavities were turned off here, but simply stated, BMAD defines a region to begin at the beginning of a cryomodule whereas LIAR defines it to begin at a BPM. The last turned off cavity in both codes was upstream of the beginning of their respective region. The selection of 


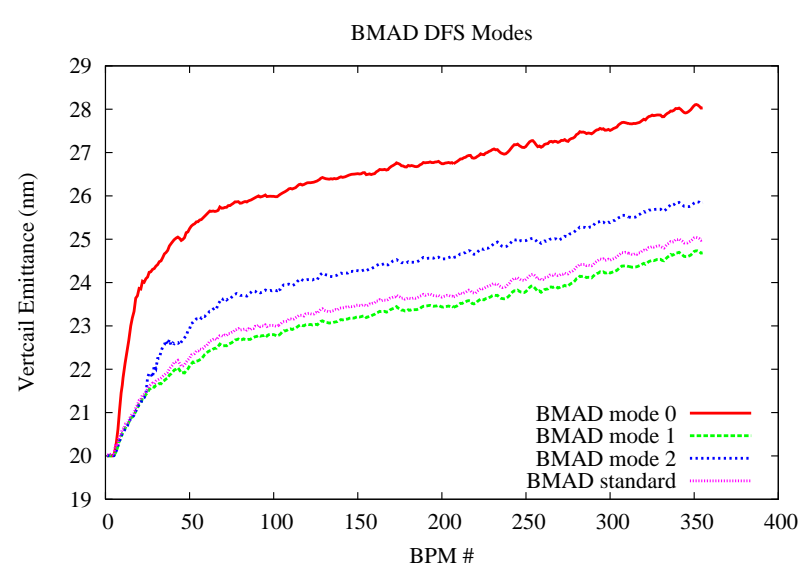

Figure 4: BMAD's 3 modes plus the "standard" mode.

cavities turned off provided the most significant difference in performance between the two codes.

\section{Detailed Study Between BMAD and SLEPT'S Three Modes.}

SLEPT and BMAD have three "modes" of DFS. The main difference between these three modes and the "standard" DFS method is that the modes will scale all accelerating cavities by a set amount versus turning off cavities. The critical differences between the three modes are summarized in [14]. The agreement between BMAD's and SLEPT's three modes is good. Figure 4 gives the three modes plus the "standard" mode, all simulated in BMAD. Mode 1 performs the best and is virtually identical to the "standard" mode. Mode 2 begins to diverge at the beginning of the second DFS region suggesting that the resteering is important, though not critical. It should be noted that if the "standard" mode is performed with no resteering, the curve is completely off-scale and fails to perform at even remotely acceptable levels. Scaling all cavities (versus turning some of them off) reduces the incoming orbit change for the off-energy beam, so re-steering is not as necessary. Mode 0 is clearly the least effective and emphasizes the added effects of the cavity pitches which are not corrected with this mode.

Mode 2 is appealing because it is the simplest algorithm and yet the degradation in performance over the "standard" mode is very small. Removing the re-steering step will improve sensitivity studies. BPM resolution is of particular concern since the effectiveness of re-steering is highly dependent on the BPM resolution.

\section{CONCLUSIONS}

Studies \#1 and \#2 demonstrate that emittance dilution behaves very similarly between five of the ILC simulation codes. Each utilizes a different beam representation which was not found to affect the results. Study \#3 shows that each code can reproduce each others' results when per- forming DFS. However, there were several simplifications with this study that may ignore potential differences between the codes.

Once the baseline lattice is well established another set of comparisons with the new lattice would be beneficial. Nevertheless, the studies performed so far demonstrate that each code can reproduce the others' results for an ILC-like lattice. There is therefore now a "benchmark" for Dispersion Free Steering, in the context of the ILC main linac, that newer codes can be compared to when beginning ILC emittance preservation work. The importance of these comparisons cannot be overstated. Beam-Based Alignment cannot be directly tested experimentally before the construction of the ILC, only simulations can be performed.

\section{REFERENCES}

[1] D. Schulte. et al., "Tests of 3 Linear Collider Beam Dynamics Simulation Programs," LCC-0091, Tesla-2002-08, CLIC-513, (2002)

[2] Andrej Wolski and Winfried Decking, "Comparison of Emittance Tuning Simulations in the NLC and TESLA Damping Rings," LCC-010, TESLA-2002-10, CBP-TechNote-268 (2002)

[3] S. Redaelli et al., "Comparison of different tracking codes for Beam Delivery Systems of Linear Colliders," Proceedings European Particle Accelerator Conference, MOPRI050 (2002)

[4] J. Urban, et al., "Linear Accelerator Simulations with Bmad," Proc. U.S. Particle Accelerator Conference, RPP026 (2005)

[5] http://www. lepp.cornell.edu/ dcs/bmad

[6] http://www.slac.stanford.edu/accel/ilc/codes/ liar/web/liar.htm

[7] http://www.desy.de/ merlin

[8] http://savannah.cern.ch/projects/placet/

[9] http://mad.web.cern.ch/mad

[10] J. C. Smith et al., "Benchmarking/Crosschecking DFS in the ILC Main Linac,” SLAC-TN-06-035 (2006)

[11] Ostiguy, J. F., "CHEF: A Framework for Accelerator Optics and Simulation," International Computational Accelerator Physics Conference, TUAPMP02 (2006)

[12] http://www.slac.stanford.edu/accel/ilc/codes/ Lucretia/

[13] http://lcdev.kek.jp/ kkubo/reports/MainLinacsimulation/SLEPT/SLEPT-index.html

[14] J. C. Smith, "The Preservation of Emittance and Polarization in the International Linear Collider," Ph.D. thesis, to be published (2007)

[15] P. Eliasson et al., "Study of an ILC Main Linac that Follows the Earth Curvature," Proceedings European Particle Accelerator Conference, MOPLS098 (2006) 